Applications of the CE/SE Scheme to Incompressible Viscous Flows in Two-Sided Lid-Driven Square Cavities

This article has been downloaded from IOPscience. Please scroll down to see the full text article.

2012 Chinese Phys. Lett. 29084707

(http://iopscience.iop.org/0256-307X/29/8/084707)

View the table of contents for this issue, or go to the journal homepage for more

Download details:

IP Address: 159.226.231.80

The article was downloaded on 11/12/2012 at 08:50

Please note that terms and conditions apply. 


\title{
Applications of the CE/SE Scheme to Incompressible Viscous Flows in Two-Sided Lid-Driven Square Cavities*
}

\author{
YANG Duo-Xing(杨多兴 $)^{1}$, ZHANG De-Liang(张德良) $)^{2 * *}$ \\ ${ }^{1}$ Institute of Crustal Dynamics, Chinese Earthquake Administration, Beijing 100085 \\ ${ }^{2}$ LHD, Institute of Mechanics, Chinese Academy of Sciences, Beijing 100190
}

(Received 1 April 2012)

\begin{abstract}
The spacetime conservation element-solution element (CE/SE) method is extended to two-dimensional incompressible viscous flow in a two-sided lid-driven square cavity. Based on the SIMPLE method concept, the preconditioned dual-time scheme is introduced for unsteady computations. The CE/SE-based code is validated by simulating one-sided lid-driven cavity flows. The two-sided lid-driven square cavity problem involves several interesting characteristics being successfully predicted, including development of a pair of off-corner vortices and a free shear layer in the case of parallel wall motion, as well as the appearance of corner vortices for lower Reynolds numbers in the case of anti-parallel motion of the walls. It is found that both the Reynolds number and the direction of the moving walls affect the fluid flow in the cavity.
\end{abstract}

PACS: 47.40.Rs, 02.60.Cb, 82.33.Vx DOI: $10.1088 / 0256-307 \mathrm{X} / 29 / 8 / 084707$

The lid-driven cavity flow problem, involving almost all fluid mechanical phenomena in the simplest geometrical settings,,$^{[1]}$ is of great scientific interest. Ghia et al. ${ }^{[2]}$ used a multi-grid method to investigate the flow field in a lid-driven cavity. Recently, two-lid driven cavity flow problems have been experimentally ${ }^{[3]}$ and numerically ${ }^{[4-6]}$ investigated.

The spacetime conservation element and solution element (CE/SE) method, first proposed by Chang, ${ }^{[7]}$ has been used to obtain highly accurate numerical solutions for hyperbolic conservation equations. The basic idea of the $\mathrm{CE} / \mathrm{SE}$ method is global and local flux conservation in a spacetime domain. Due to its efficiency, simplicity, and accuracy, the $\mathrm{CE} / \mathrm{SE}$ method has been successfully applied to viscous flow problems. ${ }^{[8-11]}$

In this Letter, based on the SIMPLE method concept, the preconditioned dual-time scheme ${ }^{[12]}$ is introduced. The CE/SE method proposed by Wang et $a l .{ }^{[13]}$ is extended to two-sided lid-driven cavity flows.

The basic equations governing the flow of an incompressible viscous fluid are the conservation of mass, i.e.,

$$
\nabla \cdot \boldsymbol{u}=0
$$

and conservation of momentum, i.e.,

$$
\frac{\partial \boldsymbol{u}}{\partial t}+\nabla \cdot(\boldsymbol{u} u)=-\frac{1}{\rho} \nabla p+\frac{1}{\rho R_{e}} \nabla \cdot \mu\left(\nabla \boldsymbol{u}+(\nabla \boldsymbol{u})^{T}\right)
$$

where $\boldsymbol{u}$ represents the fluid velocity vector; $p$ and $\rho$ are, respectively, the pressure and density of the fluid; $\mu$ denotes the dynamic viscosity; $R_{e}$ is the Reynolds number, with $R_{e}=\rho L U / \mu$. $L$ and $U$ represent, respectively, the appropriate length and velocity scale.

Next, details of the CE/SE framework used in the development of the incompressible viscous flow solver are described. The governing Eqs. (1) and (2) can be expressed as the Euler equation

$$
\frac{\partial \boldsymbol{Q}}{\partial t}+\frac{\partial \boldsymbol{E}(\boldsymbol{Q})}{\partial x}+\frac{\partial \boldsymbol{F}(\boldsymbol{Q})}{\partial y}=0,
$$

where $\boldsymbol{Q}, \boldsymbol{E}, \boldsymbol{F}$ are vectors of primary variable, flux in $x$-direction and flux in $y$-direction, respectively. Wang et al. ${ }^{[13]}$ proposed an improved CE/SE method with first-order accuracy by adopting a general hexahedron mesh to construct CEs and SEs, which is different to Chang's original CE/SE method ${ }^{[7]}$ (see Fig. 1). Let $(j, k, n)$ denote a set of spacetime mesh points, where $n=0, \pm 1 / 2, \pm 1, \pm 3 / 2, \ldots$ for time, $j=0, \pm \frac{1}{2}, \pm 1, \pm 3 / 2, \ldots$ for $x, k=$ $0, \pm 1 / 2, \pm 1, \pm 3 / 2, \ldots$ for $y$. An SE is defined as the vicinity of a mesh point and the whole spacetime region is divided into non-overlapping CEs. Assume that the physical variables in every SE are approximated by Taylor's expansions at the mesh point associated with the SE, and the conservation Eq. (3) is satisfied in every CE.

Let $x_{1}=x, x_{2}=y, x_{3}=t$ be considered as the coordinates of a Euclidean space $E_{3}$. By means of Gauss' divergence theorem, Eq. (3) can be rewritten as

$$
\oint_{S(V)} \boldsymbol{H}_{m} \cdot d \boldsymbol{s}=\int_{V} S_{m} d V
$$

where $\boldsymbol{H}_{m}=\left(E_{m}, F_{m}, Q_{m}\right)$ is the spacetime flux vector with $Q_{m}, E_{m}$ and $F_{m}$ being the components of vectors $\boldsymbol{Q}, \boldsymbol{E}$ and $\boldsymbol{F} ; S_{m}$ represents the components of the source term vector; $S(V)$ is the boundary of an arbitrary spacetime region $V$ in $E_{3}, d \boldsymbol{s}=d \sigma \hat{\boldsymbol{n}}$ with $d \sigma$ and $\hat{\boldsymbol{n}}$, respectively, being the area and the outward unit normal of a surface element on $S(V)$. Figure 1(a) shows the projection of mesh points on the $x-y$ plane, in which the interval between the mesh points denoted by closed and open circles is $\Delta t / 2$ in the time direction or $1 / 2$ in the mesh number $n$. For any point $P^{\prime}(j, k, n)$ on which the variables are solved, we define the solu-

*Supported by the Institute of Crustal Dynamics, CEA under Grant No ZDJ2010-26, and Wenchuan Fault Scientific Drilling (WFSD-10).

** Corresponding author. Email: dlzhang@imech.ac.cn

(C) 2012 Chinese Physical Society and IOP Publishing Ltd 
tion element $\mathrm{SE}\left(P^{\prime}\right)$ constituted by the three vertical planes intersecting at $P^{\prime}(j, k, n)$ and their neighborhood space as demonstrated in Fig. 1(b). Suppose that $Q_{m}, E_{m}$ and $F_{m}$ at point $(t, x, y)$ in $S E\left(P^{\prime}\right)$ are approximated by the first-order Taylor expansions at $P^{\prime}(j, k, n)$, i.e.,

$$
\begin{aligned}
Q_{m}(d x, d y, d t)_{P^{\prime}}= & \left(Q_{m}\right)_{P^{\prime}}+\left(Q_{m x}\right)_{P^{\prime}} d x+\left(Q_{m y}\right)_{P^{\prime}} d y \\
& +\left(Q_{m t}\right)_{P^{\prime}} d t, \\
E_{m}(d x, d y, d t)_{P^{\prime}}= & \left(E_{m}\right)_{P^{\prime}}+\left(E_{m x}\right)_{P^{\prime}} d x+\left(E_{m y}\right)_{P^{\prime}} d y \\
& +\left(E_{m t}\right)_{P^{\prime}} d t, \\
F_{m}(d x, d y, d t)_{P^{\prime}}= & \left(F_{m}\right)_{P^{\prime}}+\left(F_{m x}\right)_{P^{\prime}} d x+\left(F_{m y}\right)_{P^{\prime}} d y \\
& +\left(F_{m t}\right)_{P^{\prime}} d t,
\end{aligned}
$$

where $d x=x-x_{P^{\prime}}, d y=y-y_{P^{\prime}}, d t=t-t_{P^{\prime}}$, where $x_{p^{\prime}}, y_{p^{\prime}}$ and $t_{p^{\prime}}$ are the position coordinates of point $P^{\prime}$. Substituting Eq. (5) into Eq. (3) gives

$$
\left(Q_{m t}\right)_{P^{\prime}}=-\left(E_{m x}\right)_{P^{\prime}}-\left(F_{m y}\right)_{P^{\prime}}
$$
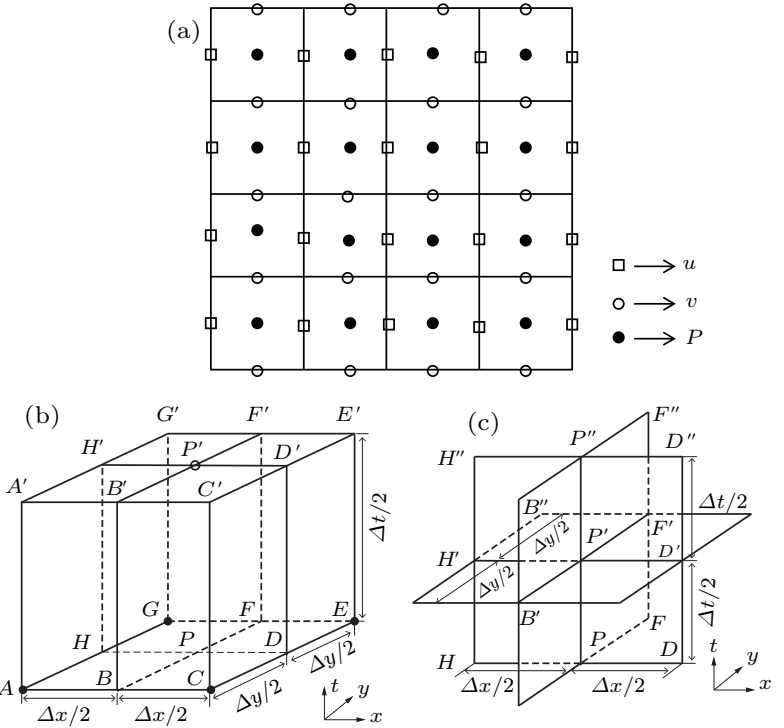

Fig. 1. Staggered spacetime mesh of the CE/SE scheme.

The above equations imply that the variables required in computation are $\left(Q_{m}\right)_{P^{\prime}},\left(Q_{m x}\right)_{P^{\prime}}$ and $\left(Q_{m y}\right)_{P^{\prime}}$, because $E_{m}$ and $F_{m}$ are the functions of $Q_{m}$. The conservation element $\mathrm{CE}\left(P^{\prime}\right)$ is defined as illustrated as Fig. 1(c). It can be seen from Fig. 1(c) that $\mathrm{CE}\left(P^{\prime}\right)$ is related to not only $\mathrm{SE}\left(P^{\prime}\right)$ but also the $\operatorname{SEs}$ of $\operatorname{SE}(A), \operatorname{SE}(C), \operatorname{SE}(E)$, and $\operatorname{SE}(G)$. Note that the values of physical variables on mesh points $A, C, E$, and $G$ are known. Assume that the integral conservation laws are satisfied in every $\mathrm{CE}$. Integrating Eq. (4) on the surfaces of $\mathrm{CE}\left(P^{\prime}\right)$ with the aid of Eq. (6), we find

$$
\left(Q_{m}\right)_{P^{\prime}}=\frac{1}{4}\left(\bar{Q}+\frac{\Delta t}{\Delta x} \bar{E}+\frac{\Delta t}{\Delta y} \bar{F}\right),
$$

where

$$
\begin{aligned}
\bar{Q}= & Q_{m}\left(A, \frac{\Delta x}{4}, \frac{\Delta y}{4}, 0\right)+Q_{m}\left(C,-\frac{\Delta x}{4}, \frac{\Delta y}{4}, 0\right) \\
& +Q_{m}\left(E,-\frac{\Delta x}{4},-\frac{\Delta y}{4}, 0\right) \\
& +Q_{m}\left(G, \frac{\Delta x}{4},-\frac{\Delta y}{4}, 0\right), \\
\bar{E}= & E_{m}\left(A, 0, \frac{\Delta y}{4}, \frac{\Delta t}{4}\right)-E_{m}\left(C, 0, \frac{\Delta y}{4}, \frac{\Delta t}{4}\right) \\
& -E_{m}\left(E, 0,-\frac{\Delta y}{4}, \frac{\Delta t}{4}\right)+E_{m}\left(G, 0,-\frac{\Delta y}{4}, \frac{\Delta t}{4}\right), \\
\bar{F}= & F_{m}\left(A, \frac{\Delta x}{4}, 0, \frac{\Delta t}{4}\right)+F_{m}\left(C,-\frac{\Delta x}{4}, 0, \frac{\Delta t}{4}\right) \\
& -F_{m}\left(E,-\frac{\Delta x}{4}, 0, \frac{\Delta t}{4}\right)-F_{m}\left(G, \frac{\Delta x}{4}, 0, \frac{\Delta t}{4}\right) .
\end{aligned}
$$

Using the continuity conditions at points $A^{\prime}, C^{\prime}, E^{\prime}$ and $G^{\prime}$, the derivatives of $Q_{m}$ with respect to $x$ and $y$ are obtained,

$$
\begin{aligned}
& \left(Q_{x}\right)_{P^{\prime}}=W\left[\left(Q_{x}\right)_{P^{\prime}}^{-},\left(Q_{x}\right)_{P^{\prime}}^{+}, \alpha\right], \\
& \left(Q_{y}\right)_{P^{\prime}}=W\left[\left(Q_{y}\right)_{P^{\prime}}^{-},\left(Q_{y}\right)_{P^{\prime}}^{+}, \alpha\right],
\end{aligned}
$$

where

$$
\begin{aligned}
\left(Q_{m x}\right)_{D^{\prime}}= & {\left[Q_{m x}(C, 0,0, \Delta t / 2)\right.} \\
& \left.+Q_{m x}(E, 0,0, \Delta t / 2)\right] / 2 \\
\left(Q_{m x}\right)_{H^{\prime}}= & {\left[Q_{m x}(G, 0,0, \Delta t / 2)\right.} \\
& \left.+Q_{m x}(A, 0,0, \Delta t / 2)\right] / 2 \\
\left(Q_{m y}\right)_{F^{\prime}}= & {\left[Q_{m y}(E, 0,0, \Delta t / 2)\right.} \\
& \left.+Q_{m y}(G, 0,0, \Delta t / 2)\right] / 2 \\
\left(Q_{m y}\right)_{B^{\prime}}= & {\left[Q_{m y}(A, 0,0, \Delta t / 2)\right.} \\
& \left.+Q_{m y}(C, 0,0, \Delta t / 2)\right] / 2
\end{aligned}
$$

Here $\left(Q_{x}\right)_{P^{\prime}}^{ \pm}$and $\left(Q_{y}\right)_{P^{\prime}}^{ \pm}$are defined as

$$
\begin{aligned}
\left(Q_{m x}\right)_{P^{\prime}}^{-}= & -\frac{1}{\Delta x}\left[Q_{m}\left(A, 0,0, \frac{\Delta t}{2}\right)\right. \\
& \left.+Q_{m}\left(G, 0,0, \frac{\Delta t}{2}\right)-2\left(Q_{m}\right)_{P^{\prime}}\right], \\
\left(Q_{m x}\right)_{P^{\prime}}^{+}= & +\frac{1}{\Delta x}\left[Q_{m}\left(C, 0,0, \frac{\Delta t}{2}\right)\right. \\
& \left.+Q_{m}\left(E, 0,0, \frac{\Delta t}{2}\right)-2\left(Q_{m}\right)_{P^{\prime}}\right], \\
\left(Q_{m y}\right)_{P^{\prime}}^{-}= & -\frac{1}{\Delta y}\left[Q_{m}\left(A, 0,0, \frac{\Delta t}{2}\right)\right. \\
& \left.+Q_{m}\left(C, 0,0, \frac{\Delta t}{2}\right)-2\left(Q_{m}\right)_{P^{\prime}}\right], \\
\left(Q_{m y}\right)_{P^{\prime}}^{+}= & +\frac{1}{\Delta y}\left[Q m\left(E, 0,0, \frac{\Delta t}{2}\right)\right. \\
& \left.+Q_{m}\left(G, 0,0, \frac{\Delta t}{2}\right)-2\left(Q_{m}\right)_{P^{\prime}}\right] .
\end{aligned}
$$

The weighted equation ${ }^{[14,15]}$ is

$$
W\left[x_{+}, x_{-}, \alpha\right]=\frac{\left|x_{+}\right|^{\alpha} x_{-}+\left|x_{-}\right|^{\alpha} x_{+}}{\left|x_{-}\right|^{\alpha}+\left|x_{-}\right|^{\alpha}},
$$


and $\alpha$ is a constant ( $\alpha=2$ in this study).

Based on the SIMPLE method concept, the preconditioned dual-time scheme ${ }^{[12]}$ is introduced. Equation (1) can be rewritten as

$$
\frac{\partial P}{\partial \tau}+C^{2} \rho\left(\frac{\partial u}{\partial x}+\frac{\partial v}{\partial y}\right)=0
$$

where $\tau$ is the visual time, and $C^{2}$ is the coefficient effecting the numerical stability. Substituting the visual time derivative of velocities into Eq. (2), we obtain

$$
\frac{\partial U}{\partial \tau}+\frac{\partial Q}{\partial t}+\frac{\partial E(Q)}{\partial x}+\frac{\partial F(Q)}{\partial y}=0 .
$$

For $\tau \rightarrow \infty$, Eq. (14) is consistent to Eq. (2). Applying the pressure splitting method, Eq. (14) takes the form

$$
\frac{\partial U_{v}}{\partial \tau}+\frac{\partial Q_{v}}{\partial t}+R_{v}\left(Q_{v}\right)+\frac{1}{\rho} \nabla P=0,
$$

where

$$
\begin{gathered}
R_{v}\left(Q_{v}\right)=\left(\frac{\partial E_{v}\left(Q_{v}\right)}{\partial x}+\frac{\partial F_{v}\left(Q_{v}\right)}{\partial y}\right), \\
Q_{v}=U_{v}=\left(\begin{array}{c}
u \\
v
\end{array}\right), \quad U=\left(\begin{array}{c}
P / C^{2} \\
u \\
v
\end{array}\right), \\
E_{v}=\left(\begin{array}{c}
u^{2}-\frac{\tau_{x x}}{\rho R_{e}} \\
u v-\frac{\tau_{y x}}{\rho R_{e}}
\end{array}\right), \quad F_{v}=\left(\begin{array}{c}
u v-\frac{\tau_{x y}}{\rho R_{e}} \\
v^{2}-\frac{\tau_{y y}}{\rho R_{e}}
\end{array}\right) .
\end{gathered}
$$

Using the time operator splitting method to split pressure item in momentum Eq. (15), we obtain

$$
\begin{aligned}
& \frac{Q_{v}^{n+1 / 2}-f\left(Q_{v}^{n}\right)}{\Delta t}+R_{v}^{n}\left(Q_{v}^{n}\right)=0, \\
& \frac{\partial U}{\partial \tau}+\frac{Q_{v}^{n+1}-Q_{v}^{n+1 / 2}}{\Delta t}+\frac{1}{\rho} \nabla P=0 .
\end{aligned}
$$

We interpret Eq. (16a) as yielding an intermediate value of $Q_{v}^{n}$, denoted by $Q_{v}^{n+1 / 2}$ at time $n$. Here the superscript $n$ indicates the time step. $F\left(Q_{v}^{n}\right)$ is the value of $Q_{v}^{n}$ at time step $n$. Equation (16a) can be simulated by using the $\mathrm{CE} / \mathrm{SE}$ method to obtain $Q_{v}^{n+1 / 2}$. By means of time-marching solutions and internal iteration method, Eqs. (13) and (16b) yield

$$
\begin{aligned}
& \frac{P^{m+1}-f\left(P^{m}\right)}{\Delta \tau}+C^{2}\left(\frac{\partial u}{\partial x}+\frac{\partial v}{\partial y}\right)^{m}=0, \\
& \frac{U^{m+1}-U^{m}}{\Delta \tau}+\frac{Q^{m+1}-Q^{n+1 / 2}}{\Delta t}+\frac{1}{\rho} \nabla P^{m+1}=0,
\end{aligned}
$$

where $m$ is the iteration step. Assume that we have already carried out $m$ iteration. Then, for the $m+1$ iteration, by substituting $Q_{v}^{n+1 / 2}$ into Eq. (17a) and using the CE/SE method, $P^{m+1}$ can be calculated at iteration step $m+1$. Substituting $P^{m+1}$ into Eq. (17b), $U^{m+1}$ is obtained. The above procedure is repeated for a number of iterations; convergence is achieved when $U^{m+1}-U^{m}$ becomes less than a prescribed value $\left(10^{-6}\right)$ at all grid points. After convergence, $U^{n+1}$ can be obtained at time step $n+1$. These governing equations are discretized on staggered orthogonal grids, which eliminate the possibility of a checkerboard pressure pattern.

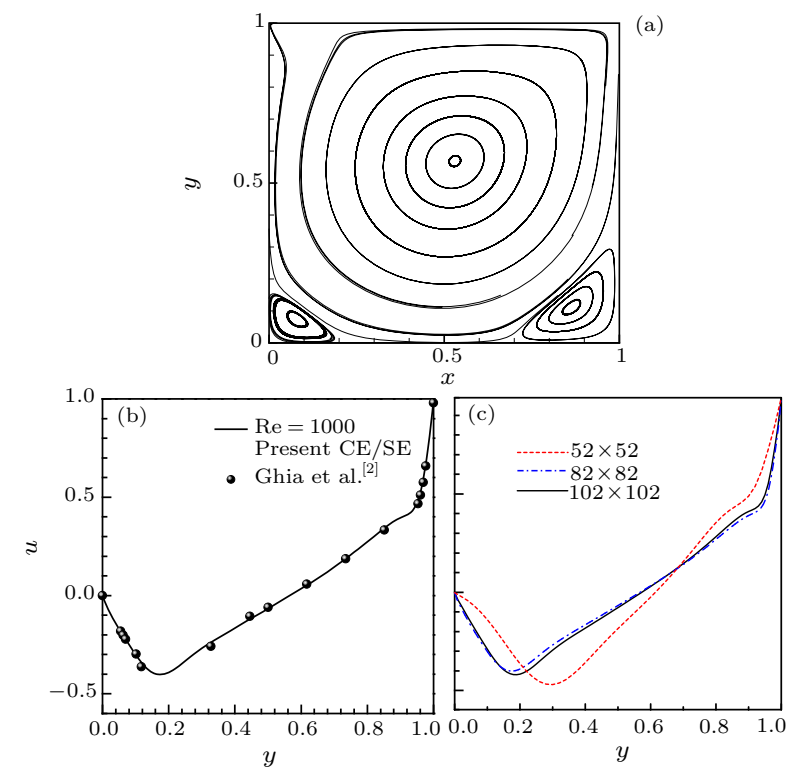

Fig. 2. Code validation: (a) streamlines $(102 \times 102$ grids $)$, (b) $u$ along the vertical centerline, and (c) grid independence test: $u$-velocity distribution in the vertical midplane $\left(R_{e}=1000\right)$.
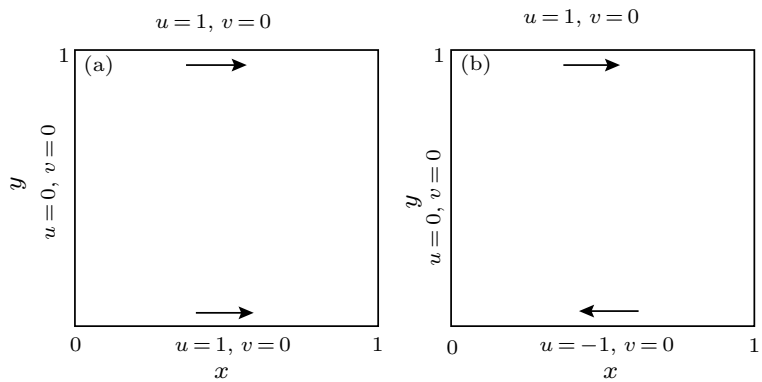

Fig. 3. Two-sided lid-driven cavity: (a) parallel wall motion and (b) anti-parallel wall motion.

In order to validate the developed $2 \mathrm{D}$ incompressible viscous flow algorithm, the code is applied to the single lid-driven cavity flow problem. Here a set of grids $(102 \times 102)$ is used, and we set $R_{e}=1000$. Qualitative comparison is shown in Fig. 2. There is a primary vortex inside the cavity, and two smaller counter-rotating vortices appear at both corners of the bottom wall. One can see an excellent agreement between the $\mathrm{CE} / \mathrm{SE}$ solutions and the benchmark solutions ${ }^{[2]}$ for the cases considered.

Figure 2(b) shows the $u$ velocity distribution along the vertical centerline, compared with Ghia's data. It can be seen that the result matches very well with Ghia's. Note that a $128 \times 128$ mesh was used in Ghia's work. It demonstrates that the $\mathrm{CE} / \mathrm{SE}$ method can be extended to $2 \mathrm{D}$ incompressible viscous flow with- 
out increasing computational effort or compromising the accuracy.

Grid convergence study is also carried out for this test case. Grid independence analysis is performed using successively sized grids, $52 \times 52,82 \times 82,102 \times 102$ for the case. ${ }^{[2]}$ Uniform grid has been used for all the computations. The distribution of the $u$-velocity in the vertical mid-plane is shown in Fig. 2(c). It is observed that the curves overlap with each other for $102 \times 102$ and $82 \times 82$. Thus a grid number of $82 \times 82$ is chosen for further computation. A similar type of grid independence test has been carried out for other cases and is not represented here.

Here we focus on the recirculating flow due to a moving lid on a cavity, and present the evolution of recirculating cells for two cases according to the top and bottom walls moving in the opposite (anti-parallel motion) or same (parallel motion) direction with a unite velocity. The boundary conditions for parallel and anti-parallel wall motion problems are illustrated in Figs. 3(a) and 3(b), respectively.
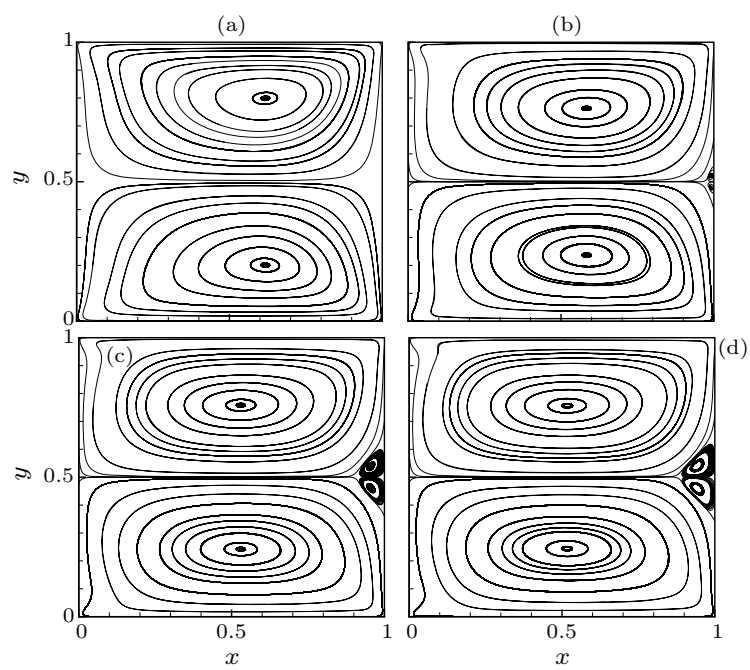

Fig. 4. Streamlines for parallel wall motion at (a) $R_{e}=$ 100, (b) $R_{e}=400$, (c) $R_{e}=1000$, and (d) $R_{e}=2000$ on $82 \times 82$ grids.
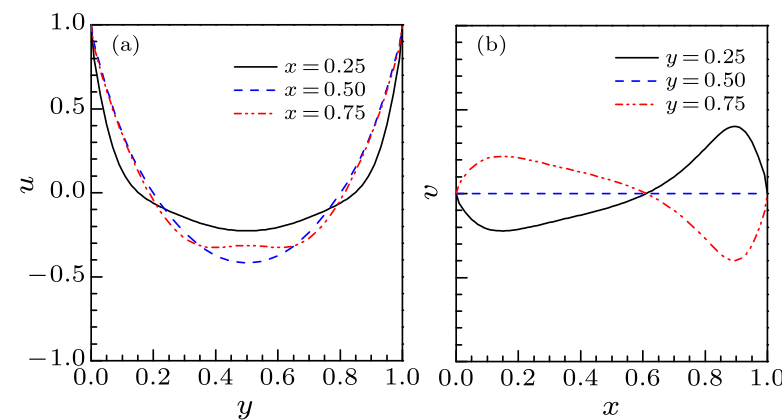

Fig. 5. Parallel wall motion at $R_{e}=100$ : (a) horizontal velocity $u$ along vertical lines $(x=0.25,0.50,0.75)$ and (b) vertical velocity $v$ along horizontal lines $(y=0.25$, $0.50,0.75)$.

Figure 4 depicts the streamlines calculated with $82 \times 82$ grids for $R_{e}=100,400,1000$ and 2000 . The upper and lower walls move in the same direction along the $x$ axis with the same velocity. The streamlines are symmetrical with respect to a line parallel to these walls and passing through the cavity center. Figure 4(a) illustrates the streamlines in the case of the parallel wall motion at $R_{e}=100$ with the top and bottom walls moving from the left to right. We observe that a pair of recirculating cells takes place at the upper and the lower cavities. These primary vortices are counter-rotating and form a typically dual lid-driven cavity flow. Clearly, there is a strong shear layer in a region of clustering stream lines between the upper and lower cavities.
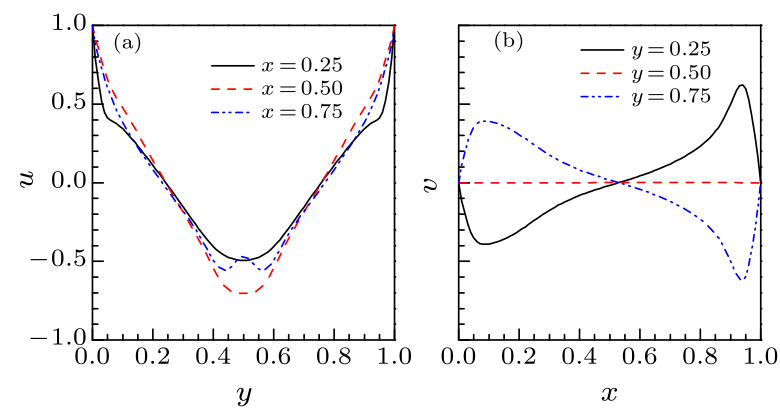

Fig. 6. Parallel wall motion at $R_{e}=1000$ : (a) horizontal velocity $u$ along vertical lines $(x=0.25,0.50,0.75)$ and (b) vertical velocity $v$ along horizontal lines $(y=0.25$, $0.50,0.75)$.
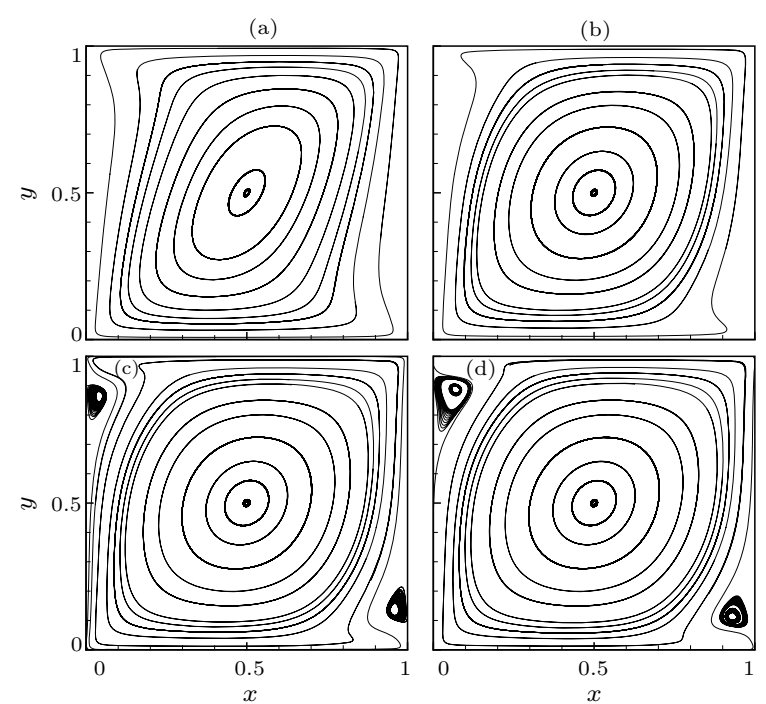

Fig. 7. Streamlines in the case of anti-parallel wall motion at (a) $R_{e}=100$, (b) $R_{e}=400$, (c) $R_{e}=1000$, and (d) $R_{e}=2000$ on $82 \times 82$ grids.

For $R_{e}=400$ as shown in Fig. 4(b), besides the primary vortices, a pair of counter-rotating secondary vortices symmetrically exist near the center of the right wall. Figures $4(\mathrm{c})$ and $4(\mathrm{~d})$ show the streamline patterns at $R_{e}=1000$ and $R_{e}=2000$. As the Reynolds number increases, the upper eddy strength and its center location move downward to the center of the upper cavity. The location of the lower eddy shifts upward to the center of the lower cavity. A free shear layer exists midway between the upper and lower cavities, referencing to the confining zone.

As the Reynolds number increases, the size of the 
secondary vortices near the center of the right wall increases. Pairs of the counter-rotating primary and secondary vortices maintain their symmetry at all the Reynolds numbers considered here.
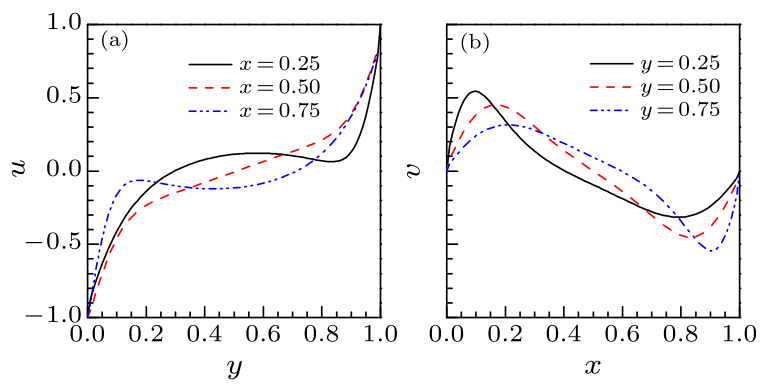

Fig. 8. Anti-parallel wall motion at $R_{e}=100$ : (a) horizontal velocity $u$ along vertical lines $(x=0.25,0.50,0.75)$ and (b) vertical velocity $v$ along horizontal lines $(y=0.25$, $0.50,0.75)$.

Figures 5 and 6 present the plots of horizontal velocity profiles along vertical lines and vertical velocity profiles along horizontal lines passing through different points of the square cavity at a variety of Reynolds numbers.

Figure 7 shows the streamline patterns simulated on $82 \times 82$ grids for $R_{e}=100,400,1000$ and 2000 . Here the upper and lower walls move in opposite directions along the $x$-axis with the same velocity. Figures $7(\mathrm{a})$ and $7(\mathrm{~b})$, respectively, depict the streamlines for $R_{e}=100$ and 400 . It is observed that a single primary vortex appears at the geometric center of the cavity for low Reynolds numbers. Figures 7(c) and 7(d), respectively, illustrate the streamline patterns for $R_{e}=1000$ and 2000. Two secondary vortices exist near the top left and the bottom right corners of the cavity, and the primary vortex center slightly shifts from the geometric center of the cavity. As the Reynolds number (between $R_{e}=1000$ and 2000) increases, the size of the secondary vortices increases. Figures 8 and 9 show the plots of horizontal velocity profiles along vertical lines and vertical velocity profiles along horizontal lines passing through different points of the square cavity at different Reynolds numbers. Also note that for all lid-driven cavity flows, there exist singularities at the corners where the velocity becomes discontinuous, while the stream function itself does not have any singularity.

Table 1. Locations of the vortices for parallel wall motions for $R_{e}=400$. Here superscript B represents the bottom and $\mathrm{T}$ denotes the top.

\begin{tabular}{lcccc}
\hline \multicolumn{3}{c}{ Primary vortex } & Secondary vortices \\
\hline Ref. [6] & $(0.5845,0.2388)^{\mathrm{B}}$ & $(0.5845,0.7553)^{\mathrm{T}}$ & $(0.9873,0.4638)^{\mathrm{B}}$ & $(0.9873,0.5264)^{\mathrm{T}}$ \\
CE/SE & $(0.5875,0.2375)^{\mathrm{B}}$ & $(0.5875,0.7500)^{\mathrm{T}}$ & $(0.9875,0.4625)^{\mathrm{B}}$ & $(0.9875,0.5250)^{\mathrm{T}}$ \\
\hline
\end{tabular}
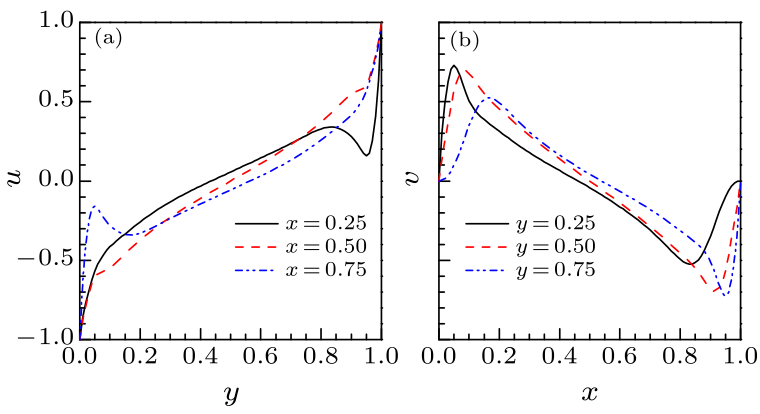

Fig. 9. Anti-parallel wall motion at $R_{e}=1000$ : (a) horizontal velocity $u$ along vertical lines $(x=0.25,0.50,0.75)$ and (b) vertical velocity $v$ along horizontal lines $(y=0.25$, $0.50,0.75)$.

Table 2. Locations of the vortices for anti-parallel wall motions for $R_{e}=400$. Here superscript BR represents the bottom right and TL denotes the top left.

\begin{tabular}{ccc}
\hline & Primary vortex & Secondary vortices \\
\hline Ref. [6] & $(0.5002,0.4981)$ & $(,)^{\mathrm{BR}}(,)^{\mathrm{TL}}$ \\
CE/SE & $(0.5000,0.5000)$ & $(,)^{\mathrm{BR}}(,)^{\mathrm{TL}}$ \\
\hline
\end{tabular}

To evaluate the $\mathrm{CE} / \mathrm{SE}$ calculated results on $82 \times$ 82 grids, locations of the vortices for parallel and antiparallel wall motions are compared with those obtained in Ref. [6] on $257 \times 257$ grids for $R_{e}=400$, respectively. As shown in Tables 1 and 2, these results confirm that the results obtained through the $\mathrm{CE} / \mathrm{SE}$ solver closely match the available benchmark results.
In the present work, the flow in the two-sided lid-driven square cavity for the parallel and antiparallel wall motion is numerically investigated with the $\mathrm{CE} / \mathrm{SE}$ method on the staggered grids, using the preconditioned dual-time scheme based on the concept of the SIMPLE method. We find that the presented $\mathrm{CE} / \mathrm{SE}$ scheme is accurate for incompressible viscous flow.

\section{References}

[1] Bruneau C H and Saad M 2006 Comput. Fluid 35326

[2] Ghia U et al 1982 J. Comput. Phys. 48 (3) 387

[3] Blohm C H and Kuhlmann H C 2002 J. Fluid Mech. 450 67

[4] Albensoeder S et al 2001 Theor. Comput. Fluid Dyn. 14 223

[5] Kumar N et al 2006 The 2nd International Conference on Computational Mechanics and Simulation (Guwahati, India 8-10 December 2006) p 102

[6] Perumal D A and Dass A K 2010 CFD Lett. 213

[7] Chang S C 1995 J. Comput. Phys. 119295

[8] Venkatachari B S et al 2008 Math. Comput. Simulat. 78 653

[9] Zhang M, Yu S T and Chang S C 2004 AIAA paper 20040075

[10] Zhang M C et al 2000 The 1st International Conference on Computational Fluid Dynamics (Kyot, Japan 9-14 July 2000) p 671

[11] Guo Y H et al 2004 Comput. Fluid 331349

[12] Yang D X et al 2012 Trans. Porous Med. 92101

[13] Wang J T et al 2009 Comput. Fluid 38544

[14] Wang G et al 2010 Chin. Phys. Lett. 27024701

[15] Dong H F et al 2011 Chin. Phys. Lett. 28030203 